ArtefaCToS. Revista de estudios de la ciencia y la tecnología

eISSN: $1989-3612$

Vol. 9, No. 1 (2020), 2. a Época, 175-198

DOI: https://dx.doi.org/10.14201/art202091175198

\title{
La Ciencia de la Educación ante las diversas opciones metodológicas de índole disciplinar
}

\section{The Science of Education in View of the Diverse Methodological Options of a Disciplinary Nature}

\author{
Ana María ALONSO \\ Centro de Investigación de Filosofía de la Ciencia y la Tecnología, UDC, España \\ a.alonsor@udc.es
}

Recibido: 31/03/2020. Revisado: 05/04/2020. Aceptado: 06/04/2020

\section{Resumen}

Tradicionalmente, la Ciencia de la Educación - y las disciplinas en las que se diversifica - se ha clasificado entre las Ciencias Sociales. Esta caracterización es insuficiente para abordar los fenómenos educativos, que están crecientemente mediados por la Tecnología. Así, por ser hechura humana (buman made) que amplía sus posibilidades, tiene carácter dual, de modo que es también Ciencia de lo Artificial. Mediante el dominio de lo artificial, la Ciencia de la Educación es Ciencia Aplicada: utiliza diseños para resolver problemas concretos. Su estatuto como Ciencia de Diseño abre nuevas posibilidades para afrontar los problemas de la Ciencia de la Educación.

Un problema, que desafía la especificidad disciplinar, se refiere a las opciones metodológicas para atender a la complejidad, estructural y dinámica, de los fenómenos educativos. Aquí se analizan las diferentes propuestas metodológicas para superar la fragmentación del saber: la interdisciplinariedad, la multidisciplinariedad, la transdisciplinariedad y la disciplinariedad cruzada (crossdisciplinarity). Se consideran los niveles de integración que comportan. Además, por ser la Ciencia de la Educación una Ciencia Aplicada en la que la solución de los problemas atañe a la práctica en un entorno social, la integración conceptual y metodológica puede involucrar ámbitos situados más allá de la esfera académica.

Palabras clave: Ciencia de la Educación; Ciencia de lo Artificial; Ciencia de Diseño; interdisciplinariedad; multidisciplinariedad; transdisciplinariedad; disciplinariedad cruzada. 


\begin{abstract}
Traditionally, the science of education - and the disciplines in which it is diversified - has been classified among the social sciences. This characterization is insufficient to address educational phenomena, which are increasingly mediated by technology. Thus, because it is human-made, which broadens its possibilities, it has a dual character, so that it is also a science of the artificial. By means of the domain of the artificial, the science of education is Applied Science: it uses designs to solve concrete problems. Its status as a science of design opens up new possibilities for tackling the problems of the science of education.

One problem, which defies disciplinary specificity, concerns methodological options for dealing with the structural and dynamic complexity of educational phenomena. Here the different methodological proposals for overcoming the fragmentation of knowledge are analysed: interdisciplinarity, multidisciplinarity, transdisciplinarity and crossdisciplinarity. The levels of integration that they involve are considered. Furthermore, since the science of education is an applied science in which the solution of problems concerns practice in a social environment, conceptual and methodological integration may involve areas beyond the academic sphere.
\end{abstract}

Keywords: science of education; science of the artificial; science of design; interdisciplinarity; multidisciplinarity; transdisciplinarity; crossdisciplinarity.

\title{
1. La diversidad metodológica como marco conceptual
}

Hay en la Ciencia diversidad metodológica, en cuanto que estudia fenómenos y aspectos diferentes y, además, los investiga de distinta manera. La división entre las Ciencias Formales y las Ciencias Empíricas se apoya preferentemente en lo primero, aunque también reconoce lo segundo. Las diferencias en los objetos de estudio, los problemas planteados y los métodos utilizados justifican la distinción entre Ciencias de la Naturaleza, Ciencias Sociales y Ciencias de lo Artificial. Es en este tercer grupo donde se encuentran las Ciencias de Diseño (Simon, 1996), que cabe diferenciar de los estudios acerca de propiedades naturales o características sociales de lo artificial, como sucede con la Ciencia de los Materiales o la Economía de la innovación tecnológica (González, 2007, 43-44).

Al profundizar en cómo se investiga, se hace explícita una diversificación de la actividad científica entre la Ciencia Básica — que busca ampliar el conocimiento de lo real mediante explicación y predicción, para incrementar su grado de verosimilitud - y la Ciencia Aplicada, que orienta su actividad a la resolución de problemas concretos. Para ello anticipa el futuro posible, con el fin de establecer pautas de actuación orientadas a soluciones. Mientras la Ciencia Básica busca 
explicar y/o predecir, la Ciencia Aplicada necesita predicciones fiables como guía para prescribir pautas de actuación para resolver problemas ${ }^{1}$.

Cuando se especifica el tipo de conocimiento empírico investigado es cuando se llega a las Ciencias de Diseño. En ellas no se busca caracterizar cómo son las cosas sino cómo deben ser para alcanzar determinados fines, para abordar la resolución de problemas concretos. Se utilizan entonces diseños. Así, a partir de objetivos, se establecen unos procesos, para lograr unos resultados. Uno de estos ámbitos es el educativo. Por eso, metodológicamente, la Ciencia de la Educación - y las disciplinas en las que se diversifica - se sitúan primordialmente en el terreno de las Ciencias de Diseńo. Esto determina la estructura de las teorías y la orientación metodológica de estas Ciencias.

El debate acerca de la denominación disciplinar sigue abierto. En ocasiones se usa el plural "Ciencias de la Educación", tratando de resaltar el carácter interdisciplinar (o meramente multidisciplinar) de este ámbito de estudio y, otras veces, la forma singular "Ciencia de la Educación". Esta es la opción que se prefiere en este trabajo, para destacar que este campo disciplinar tiene un estatuto científico específico, que es diferente al característico de otras disciplinas con las que se relaciona e, incluso, hay relaciones de dependencia.

Desde un punto de vista metodológico, la Ciencia de la Educación engloba - a mi juicio- a la Pedagogía, si se atiende a tres razones: 1) La educación ha sido siempre su objeto de conocimiento específico, que ha buscado ampliar a través de diversos enfoques metodológicos. 2) La Pedagogía es una Ciencia Aplicada, pues su eje no está en la explicación y la predicción sino en resolver problemas concretos, de manera que combina predicción y prescripción para afrontar los problemas. 3) Usa la Pedagogía diseños para la actividad educativa, de modo que su conocimiento está relacionado con objetivos, procesos y resultados buscados. En este sentido, es una Ciencia de Diseño y tiene una dimensión artificial.

Herbert Simon (1996) insiste en asociar las Ciencias de Diseño con la dimensión de lo artificial, pues se basan en lo construido por los humanos. Inciden en esa elaboración los objetivos de los diseños, los procesos para alcanzarlos y los resultados esperables. El campo temático de los fenómenos educativos tiene - a mi juicio- esos componentes. La vertiente científica de la Educación lleva a una realidad artificial, en cuanto que está construida en razón de objetivos buscados, de modo que el diseño, para ampliar las posibilidades humanas, añade algo a la mera socialización. El carácter artificial es todavía más explícito cuando la Educación está mediada por la Tecnología. También son Ciencias de lo Artificial un conjunto de disciplinas científicas (como la Economía, la Comunicación, la Administración, etc.) que tienen una estrecha imbricación con la Educación.

\footnotetext{
${ }^{1}$ Hay un tercer tipo de investigación, centrado en cómo aplicar la Ciencia en contextos de uso concretos. Cfr. González, 2015, pp. v, 4, 18, 33, 40, 70-71, 151n, 321 y 325.
} 
Se incrementa la diversidad metodológica de la Ciencia de la Educación cuando se constata que la Pedagogía se subdivide en diferentes disciplinas, tales como la Didáctica o la Organización Escolar, de manera que hay una especialización metodológica en esos casos. Este conjunto de disciplinas conforma el campo de las Ciencias de la Educación, de modo que - a mi juicio- tienen rasgos metodológicos distintos de las Ciencias Sociales que se ocupan de los fenómenos educativos desde la perspectiva de sus disciplinas generadoras y con sus métodos propios. Esto es lo que sucede con la Psicología de la Educación, la Sociología de la Educación o la Economía de la Educación.

Conviene resaltar esta diferencia metodológica entre la Ciencia de la Educación, que engloba a la Pedagogía y sus disciplinas conexas, y las Ciencias Sociales enfocadas hacia el estudio de fenómenos educativos. Considero que este segundo conjunto de disciplinas no son Ciencias de Diseño y, además, no siempre están orientadas a realizar Ciencia Aplicada. En efecto, (i) en estas Ciencias Sociales la acción educativa no es su objeto de estudio específico; (ii) con frecuencia, no son Ciencias Aplicadas sino Ciencias Básicas, puesto que tratan muchas veces de explicar los fenómenos y realizar predicciones, pero sin plantearse la prescripción para solucionar problemas concretos; y (iii) aun cuando estas disciplinas sociales pueden generar conocimiento aplicable a fenómenos educativos, no cabe garantizar la fiabilidad de sus teorías cuando se transfieren a la Educación. Cabe afirmar que la Ciencia de la Educación — con la Pedagogía como núcleo- es una disciplina autónoma, en lugar de una rama derivada de la Psicología, la Sociología o la Economía.

Ahora bien, sucede que, tradicionalmente, se han clasificado la Ciencia de la Educación y sus diversas expresiones disciplinares entre las Ciencias Sociales. Esto es compatible con ser una Ciencia de Diseño, de manera que es metodológicamente dual. Que la Ciencia de la Educación sea una Ciencia Social se justifica a tenor de factores de índole epistemológica y ontológica: a) su objeto de estudio se desenvuelve en un medio social, b) su actividad responde a necesidades humanas (como la socialización de los niños, la transmisión de la tradición cultural, la construcción del espacio público, la base de las condiciones para el cambio social, etc.), y c) versa sobre acciones humanas intencionales que, normalmente, son propias de grupos y no suelen ser reducibles a actos aislados de individuos. Estas acciones humanas se orientan hacia fines buscados y, ciertamente, no son el resultado de la programación de una conducta vía algoritmos.

Desde el punto de vista ontológico, estamos ante una actividad humana, que despliega intencionalidad y que está situada socialmente e históricamente. En este sentido, la Educación presenta una serie de rasgos relevantes: I) su contenido se basa inicialmente en una práctica profesional, II) surge esa actividad y se desarrolla en un entorno social, y III) incide expresamente en ese medio social. Así, desde una perspectiva epistemológica, la relación es bidireccional con el entorno 
social: la actividad educativa se retroalimenta a partir de su práctica, pues la propia práctica modula la actividad educativa misma.

\section{Caracterización metodológica de la Ciencia de la Educación como Ciencia de Diseńo}

Para la caracterización metodológica como Ciencia de Diseńo, hay que partir tanto de la vertiente histórica como de la dimensión temática de la Ciencia de la Educación. Así, al analizar la configuración científica de la actividad educativa, se observa un proceso semejante al que Ilkka Niiniluoto (1993) describe en las disciplinas que derivan de la "cientificación" de las profesiones. Esa cientificación da lugar a Ciencias Aplicadas. Son disciplinas que se configuraron a partir de determinadas destrezas prácticas basadas en la experiencia, que posteriormente se concretaron en reglas de acción y se sistematizaron, dando lugar a un cuerpo organizado de conocimiento que permite resolver problemas concretos. Cuando el funcionamiento de estas reglas comienza a validarse mediante pruebas científicas, emerge entonces una Ciencia de Diseño.

Cuando se "cientifica" el conocimiento práctico de una profesión (como la educativa), se puede dar el entrelazamiento entre la Ciencia Aplicada — la solución encontrada para los problemas concretos-y la aplicación de la Ciencia, que es el uso de ese conocimiento en un contexto concreto, dentro de unas circunstancias delimitadas espacial y temporalmente. Las soluciones propuestas por la Ciencia Aplicada miran hacia unos objetivos — solucionar algún problema-y aspiran a unos resultados. Mediante la práctica — la aplicación de la Ciencia-, que es de índole intencional, los procesos buscan la resolución efectiva de lo planteado ${ }^{2}$.

Dentro de ese marco de actividad de carácter intencional, que está dirigido a metas buscadas, el entrelazamiento entre Ciencia Aplicada y aplicación de la Ciencia resalta el papel de los diseńos educativos. Por eso, la caracterización de la Ciencia de la Educación como mera Ciencia Social resulta insuficiente y, desde luego, no permite dar cuenta de lo que supone el fenómeno educativo. Tanto la Ciencia de la Educación — su núcleo pedagógico — como sus disciplinas conexas están crecientemente mediadas por la Tecnología. Esto cambia el fenómeno educativo mismo. En la actualidad, al estar mediado ese fenómeno por la Tecnología - lo que incluye la educación a distancia en línea (online) —, hay un incremento de la dimensión artificial de la Educación. Su proyección futura solo se puede captar reconociendo la mayor relevancia de ese factor artificial.

\footnotetext{
${ }^{2}$ En ese nexo entre Ciencia Aplicada y aplicación de la Ciencia interviene habitualmente la interacción entre creatividad científica e innovación tecnológica, cfr. González (2013, 17-18).
} 


\subsection{Carácter dual de la Ciencia de la Educación: Ciencia de lo Artificial y Ciencia Social}

La Ciencia de la Educación tiene carácter dual. Es parte de las Ciencias de lo Artificial al ser hechura humana (human made). Lo es en las metas que traza, en los medios que utiliza y en los resultados que obtiene ${ }^{3}$; y se integra en las Ciencias Sociales, por las razones antes expuestas. Este estudio del carácter dual reconoce la esfera de lo social, pero resalta el ámbito de lo artificial, puesto que, preferentemente, la Ciencia de la Educación tiene el estatuto de Ciencia Aplicada de Diseño. Supondría una reducción metodológica injustificada un análisis filosófico-metodológico de la Educación realizado únicamente desde la perspectiva de las Ciencias Sociales.

Ofrecen enfoques complementarios la vertiente artificial y la dimensión social, de modo que contribuyen a dar razón de la poliédrica realidad educativa. La investigación educativa ha de atender, sin duda, a la faceta social, que cubre una parte del conjunto educativo. Esto lo ha de hacer para la resolución de problemas relacionados con la acción educativa, buscando sus posibles causas y considerando sus consecuencias. Pero, como la Ciencia de la Educación es primordialmente Ciencia Aplicada con objetivos deliberadamente buscados, prevalece la resolución de problemas concretos en el horizonte de metas. A tal efecto, la Educación se basa en diseños, que determinan objetivos, procesos y resultados ${ }^{4}$.

Es en este aspecto donde se revela insuficiente la consideración de la Ciencia de la Educación en el entorno de la Ciencia Social y se requiere la novedad de lo artificial, que potencia lo humano. I) Los diseños, en cuanto que son artificiales, pertenecen a un ámbito que no está limitado a las necesidades humanas de carácter social, sino que amplían el territorio de lo humano (González, 2008b).

\footnotetext{
${ }^{3}$ La dualidad social y artificial no es exclusiva de la Educación, sino que se trata de unos rasgos compartidos por aquellas Ciencias que están enraizadas en las necesidades humanas y en las que lo construido se superpone a lo dado. Esto sucede cuando las disciplinas investigan cómo ampliar las potencialidades humanas mediante diseńos, adentrándose entonces en un campo netamente artificial (incide aquí la influencia de las Tecnologías de la Información y la Comunicación (TIC) y los desarrollos de la Inteligencia Artificial, puesto que modulan la operatividad de nuevos objetivos, procesos y resultados). Este es el caso, de la Economía, las Ciencias de la Comunicación o las Ciencias de la Documentación, entre otras.

Parece necesario incidir en la vertiente de lo artificial, por tratarse de un enfoque que apenas ha sido abordado en Educación. Pero, además, resulta de especial interés en cuanto que existen una serie de disciplinas científicas (Economía, Ciencias de la Comunicación, Ciencias de la Documentación, Ciencias de Internet, Ciencias de la Administración, etc.) que pertenecen al ámbito de lo artificial —o bien poseen una dimensión artificial— cuyo desarrollo tiene una incidencia directa en los avances de la Educación.

${ }^{4}$ Objetivos, procesos y resultados son los elementos que caracterizan las Ciencias de Diseño en cuanto Ciencias de lo Artificial. Hay dos acepciones diferentes de Ciencias de lo Artificial: (i) las Ciencias de Diseńo que surgen a partir de prácticas profesionales y se articulan en términos de objetivos, procesos y resultados, y (ii) el estudio científico-natural o científico-social de productos o artefactos (cfr. González, 2007, 43-44).
} 
II) Además de responder a una racionalidad adaptativa, que es expresión de una racionalidad evolutiva, hay creatividad en el ámbito de lo artificial. Esto genera nuevas formas de existencia ajenas al mundo natural y lo hace en términos de historicidad, lo que propicia novedad longitudinal y transversal (González, 2012a y 2017).

Esta propuesta abre muchas posibilidades para abordar los principales problemas de la Ciencia de la Educación y podría contribuir a corregir la desorientación epistemológica que constatan los mismos estudiosos de la disciplina (cfr. Quintana Cabanas, 1983). Esa desorientación incide claramente en diversas cuestiones: a) las dudas que todavía suscita su estatuto científico; b) la polémica acerca de la denominación disciplinar: "Pedagogía", "Ciencia de la Educación" o "Ciencias de la Educación"; c) las controversias metodológicas que, a lo largo del siglo XX, han surcado a la investigación educativa en cuanto Metodología de la Ciencia de la Educación; y d) la difícil relación que tradicionalmente han mantenido la investigación en Educación y la práctica educativa.

A mi juicio, estas cuestiones se asientan generalmente sobre la base de un doble olvido: (i) no distinguir entre la investigación básica y la aplicada, y (ii) no reconocer que, dentro de las Ciencias Empíricas, están las Ciencias de Diseño como un estudio específico de lo artificial, distinto metodológicamente a las Ciencias de la Naturaleza y a las Ciencias Sociales. Pero ni los estudiosos de la Educación ni los filósofos de la Ciencia han puesto ahí el foco de atención. Así, no se han centrado en las peculiaridades de las disciplinas que se ocupan de ampliar las posibilidades humanas, de acuerdo con los nuevos retos que surgen y cambian a lo largo del tiempo 5 .

Más aún, habitualmente los análisis epistemológicos y metodológicos se han realizado en Educación considerando modelos que son más propios de las Ciencias Básicas que de la Ciencia Aplicada. Suele ser frecuente que los modelos científicos no hagan justicia a las características peculiares de la Ciencia Aplicada (cfr. Niiniluoto, 1993, 1). Esta circunstancia ha sido especialmente perjudicial para la Ciencia de la Educación, en la medida que su estatuto epistemológico se ha venido decidiendo conforme a estándares propios de ámbitos científicos que, en rigor, no son equiparables.

\footnotetext{
${ }^{5}$ La historicidad es un rasgo presente en tres niveles metodológicos: 1) la Ciencia, en general; 2) en los grupos de Ciencias; y 3) en cada Ciencia, en particular. También se encuentra en los agentes que investigan, que son seres humanos que actúan en un contexto histórico concreto, que está sujeto a variaciones. Se halla asimismo en la propia realidad investigada, sobre todo si es social o artificial (González, 2008a, 52). Esto tiene especial incidencia en el caso de las Ciencias Sociales y las Ciencias de lo Artificial, que son los campos temáticos en los que se ubica la Educación.

Hay, además, otros aspectos de historicidad: a) las relaciones de los investigadores entre sí, b) las relaciones entre los investigadores y los organismos vinculados a la investigación, y c) las relaciones de los científicos respecto del medio natural, social o artificial con el que interacciona en el curso de la investigación (González, 2017, 10-32). Estos tres tipos de relaciones son particularmente importantes en el caso de la Educación.
} 
Epistemológicamente, la Educación comporta un tipo de conocimiento distinto a la Física, la Biología o la Sociología. Así, hay un ámbito específico de la Ciencia de la Educación. Metodológicamente, las estrategias de investigación han de ser diferentes a esas u otras disciplinas. La Ciencia de la Educación tiene una doble finalidad: I) descubrir cómo se ha producido el conocimiento, para después evaluarlo, a los efectos de captar cómo se producen las acciones educativas y anticipar el futuro posible; y II) sobre la base de ese conocimiento, ha de construir las reglas de intervención pedagógica en sus propios términos, de modo que ha de predecir para poder prescribir.

El objeto de estudio que se pretende explicar o predecir es la acción pedagógica, a la que sigue la predicción y la prescripción, basada en el conocimiento científico de la actividad educativa. Ese estudio puede dar lugar a diversas interpretaciones, de las que se siguen diferentes intervenciones educativas. Las predicciones dependen de las variables conocidas y el grado de control de las variables. Las pautas de actuación que dan lugar a reglas han de ajustarse al mejor modo de intervenir en cada caso. Si hay que comprender para prescribir es porque se trabaja con diseños, sean explícitos o implícitos. Así, "el diseño como Ciencia es un instrumento tanto para comprender como para actuar" (Simon, 1996, 164).

Cuando se pone el foco en la vertiente artificial de la Educación, caracterizada como Ciencia Aplicada de Diseño, se asume lo siguiente: a) la Educación cumple el requisito de cientificación de las prácticas profesionales previas; b) dentro de un contexto teleológico, el contenido cognitivo busca una aplicación práctica, donde intervienen la predicción y la prescripción para elaborar un diseño; y c) la Educación, concebida como Ciencia, tiene una relación cada vez más frecuente con la Tecnología, en concreto, con las Tecnologías de la Información y la Comunicación. Así, por un lado, la Tecnología le sirve como soporte para determinados diseńos, y, por otro, supone un condicionante necesario para una serie de propuestas novedosas.

Mediante la cientificación de las prácticas profesionales, la Educación ha de resaltar sus peculiaridades epistemológicas y metodológicas, que están enfocadas hacia un ámbito temático que le es propio. Después, el componente del diseño resalta su versatilidad, en cuanto que la Educación se adapta a múltiples entornos posibles. Esa dimensión de lo artificial complementa la faceta de Ciencia Social: lo primero mira hacia lo "contingente", lo superfluo o lo ańadido a lo que es recibido o natural; mientras que lo segundo atiende a los factores que son necesarios para la vida humana en sociedad, aquellos de los que no se puede prescindir en modo alguno. Sus rasgos característicos son de una actividad científica dual - artificial y social—, que es distinta de las Ciencias de la Naturaleza y presentan un nexo creciente con la Tecnología. 


\subsection{Predominio de lo artificial en la Educación a partir de los diseńos}

Cuando se reconoce el papel de los diseños, parece claro el predominio de lo artificial sobre lo social en Educación. Esto se traduce en varios aspectos. (i) La Ciencia de la Educación se muestra como Ciencia de lo Artificial al buscar diseńos para resolver problemas educativos. Los diseños también propician la diversidad de modelos educativos, pues aumentan las oportunidades de las personas cuando se adentra en la esfera de lo artificial. Así, su objeto de estudio no está en la Naturaleza, sino en las nuevas formas de existencia que amplían las oportunidades humanas. Los nuevos diseños permiten afrontar nuevos retos y esas novedades "son la expresión de la historicidad de lo artificial” (González, 2018, 82).

(ii) A través del dominio de lo artificial, la Ciencia de la Educación se muestran como Ciencia Aplicada. La solución de problemas concretos atañe al conocimiento, a la actividad práctica (habilidades, destrezas, competencias, etc.) y al desarrollo de los valores. Es una búsqueda orientada y ha de lidiar con la complejidad. En un dominio práctico, donde lo teleológico es relevante, el contenido cognitivo no puede ser meramente descriptivo. Por un lado, ha de responder a los criterios de corrección, capacidad informativa y veracidad. Pero, por otro lado, su aplicabilidad exige que se evalúe en función de la simplicidad de los procesos seguidos y de los resultados obtenidos (cfr. Niiniluoto, 1993, 5-6). Hay así un componente pragmático asociado al predominio de lo artificial.

(iii) Los diseńos no miran solo ad intra, sino también ad extra. Los fines que determinan la planificación y organización de la enseñanza no se reducen a la "actividad educativa como tal", sino que han de atender a la actividad educativa como un quehacer humano entre otros. Cuando se plantea que el diseño ha de atender a cómo deberían ser las cosas, proponiendo constructos científicos que sirvan para alcanzar una meta (Simon, 1996, 114), se está abriendo la puerta a no quedarse "en lo interno" de la Educación. Así, que la Ciencia de la Educación sea Ciencia de Diseño, orientada a fines elegidos y con pautas concretas seleccionadas para alcanzar esos fines, permite repensar la meta clave de la Educación, que es - a mi juicio - la preparación para la vida en sociedad. Pero esto se concreta en formas diversas: transmitir o facilitar la adquisición de conocimientos de carácter humanístico, científico y tecnológico, formar ciudadanos, responder a las demandas del mercado y aumentar la competitividad económica, resaltar el componente ético, etc. $\left.{ }^{6}\right)$.

\footnotetext{
${ }^{6}$ Alcanzar el consenso acerca de cuál es el fin último es más sencillo que alcanzarlo acerca de su significado. Esto ya lo decía Aristóteles cuando trataba acerca del fin último de toda acción humana: "Sobre su nombre, casi todo el mundo está de acuerdo, pues tanto el vulgo como los cultos dicen que es la 'felicidad', y piensan que 'vivir bien' y 'obrar bien' es lo mismo que 'ser feliz'. Pero sobre lo que es la felicidad discuten y no lo explican del mismo modo el vulgo y los sabios" (Aristóteles, Ética Nicomaquea, I-1095a 16-21). El paralelismo con el fin que nos ocupa parece claro.
} 
Así pues, pensar la Ciencia de la Educación como Ciencia de lo Artificial requiere poner la atención en aspectos filosófico-metodológicos diferentes a los tradicionales, que las reducían a Ciencias Sociales. Porque, "considerado en términos filosófico-metodológicos, el avance en las Ciencias de lo Artificial —conceptual, práctico, social e institucional- es distinto del progreso de las Ciencias Sociales. Porque son diferentes sus objetos de estudio y sus problemas y, por ende, sus métodos, que dan lugar a resultados propios, con consecuencias de índole distinta (unas guardan relación directa con aspectos opcionales humanos, mientras que las otras están más relacionadas con necesidades humanas). Así, aun cuando las Ciencias de lo Artificial puedan analizar, buscan sobre todo sintetizar, para potenciar las posibilidades humanas en el campo de lo artificial" (cfr. González, 2018, 82).

\section{La Educación ante las diversas opciones metodológicas de índole disci- plinar}

Una de las cuestiones más debatidas en Educación se refiere a las opciones metodológicas de índole disciplinar a seguir. Así, la investigación que se realiza sobre la actividad educativa tiene una serie de características que, por un lado, le confieren especificidad y, por otro, dificultan su estudio. Estas características se relacionan con "la peculiaridad de los fenómenos que estudia, la multiplicidad de métodos que utiliza y la pluralidad de los fines y objetivos que persigue" (Arnal, Del Rincón y Latorre, 1994, 36).

Entre las peculiaridades de los fenómenos educativos figura su complejidad, que es tanto estructural como dinámica, que es interna y externa (González, 2012a). Por una parte, hay cuestiones epistemológicas y ontológicas de carácter estructural. Esto se refleja en la interacción de una diversidad de variables, muchas de las cuales - como los valores, las creencias, los significados o las intenciones - no son observables (Arnal, Del Rincón, y Latorre, 1994, 36). Son factores que, para su conocimiento, es necesario descomponer en otros más simples. Por otra parte, hay una dinámica de historicidad de los procesos educativos, que tiene diversos planos y que ciertamente dificulta su estudio.

Tanto en el plano estructural como en el dinámico, los fenómenos educativos resultantes de la interacción surgen por la intervención de diversos factores. Unos son internos a la actividad educativa misma y otros son externos. Entre esos factores, están la conducta, las relaciones sociales, la situación económica, etc. Algunos de ellos pertenecen a campos de conocimiento que no son el estrictamente educativo. De ahí que puedan ser estudiados por otras disciplinas científicas, complementando o ampliando el campo de estudio de la Ciencia de la Educación, que sería el terreno propio de la especificidad disciplinar.

Sucede, además, que ninguna de las disciplinas científicas puede, por separado, atender a las distintas variables a tener en cuenta. Porque, ante un mismo 
fenómeno educativo, puede haber enfoques distintos. Así, los factores que tiene en cuenta un sociólogo al estudiar el abandono escolar temprano son distintos a los que considera el psicólogo. En este sentido, se requiere el concurso de diversas disciplinas. Por eso, hay diversos caminos para superar la fragmentación del saber acerca de los fenómenos educativos, que un tratamiento especializado desde diferentes áreas convierte en inevitable. Tradicionalmente, se ha insistido en la interdisciplinariedad y la multidisciplinariedad. Pero progresivamente han ido ganando presencia la transdisciplinariedad y la disciplinariedad cruzada (crossdisciplinarity). Cada una de esas opciones metodológicas de índole disciplinar supone un nivel distinto de integración.

Junto a la complejidad estructural y dinámica antes mencionada, donde destacan los aspectos epistemológicos y ontólogicos, hay una dificultad añadida, que corresponde a la Semántica de la Ciencia y repercute directamente en la Metodología de la Ciencia: no hay acuerdo terminológico que tenga aceptación general ni se dispone tampoco de una tipología metodológica perfectamente perfilada acerca de tipos de interdisciplinariedad. De hecho, como señala Uskali Mäki, categorías como multi-, inter-, cruza-, pluri-, trans-, y otros tipos de X-disciplinariedad han recibido múltiples definiciones en la literatura; pero, a menudo, carecen de suficiente rigor de análisis (Mäki, 2016). Sus límites permanecen vagos, puesto que las dimensiones subyacentes no han sido elaboradas con suficiente cuidado y esto permite nuevas divisiones.

Según Mäki, los conceptos más primitivos en términos de los cuales se construyen estas tipologías incluyen la complejidad, la integración, la división de tareas de investigación, colaboración, participación, transferencia, expansión, unificación, triangulación, pluralismo y seguramente más. Son muchas las combinaciones posibles y, aunque se han sugerido una variedad de tipologías, queda todavía mucho espacio para la sofisticación conceptual, de modo que no es fácil que los esfuerzos se completen muy pronto. Por ello, considera que cualquier intento apresurado de estabilizar la terminología sería prematuro (Mäki, 2016, 331-332).

\subsection{Especificidad disciplinar}

Una primera opción metodológica es resaltar la especificidad disciplinar, que constituye el núcleo para diferenciar un tipo de disciplinas científicas — sean de la Naturaleza, Sociales o de lo Artificial - y que repercute directamente en el tipo de estudios en las Universidades (Grado, Máster, Doctorado). En este caso, la especificidad disciplinar sería de la Ciencia de la Educación en sentido estricto. Una disciplina autónoma, que ciertamente puede estar entrelazada con otras, pero que da forma al tronco de los estudios sobre la actividad educativa. A este respecto, se afirma que "es necesario construir un conocimiento propio, donde la 
explicación de los fenómenos educativos tenga significación intrínseca a la Educación" (Rodríguez Martínez, 2006, 34).

Esto supone defender un tipo de investigación que tenga en cuenta las características específicas de su objeto de estudio, que incluye el educar, el acto educativo. Es algo que atañe directamente a la dimensión artificial de la Ciencia de la Educación, que conlleva su carácter de Ciencia Aplicada y, por tanto, el estar orientada a la resolución de problemas concretos. Sin embargo, la defensa de una autonomía funcional de la Ciencia de la Educación como disciplina no es incompatible con la dependencia disciplinar o con una relación interdisciplinar, sino que es —a mi juicio— su condición de posibilidad. Porque, al igual que sucede con otras disciplinas científicas, la Ciencia de la Educación, para desarrollar su conocimiento, puede seguir dos rutas principales: a) puede utilizar conocimiento procedente de otras disciplinas, integrándolas en su acervo científico, o b) puede buscar la colaboración de otros ámbitos disciplinares, para la comprensión y solución de un problema educativo.

Planteado en estos términos, cabe metodológicamente el uso de la forma singular: la "Ciencia de la Educación". Esta expresión en singular es adecuada, como ya se señaló, "cuando lo que se quiere es resaltar que tiene un estatuto científico específico, que es diferente de otras disciplinas a partir de las cuales surge o con las que conecta" (González, 2008a, 55). En tal caso, el plural —las "Ciencias de la Educación" - se reserva para agrupar las disciplinas conexas de la Pedagogía (Organización Escolar, Orientación Escolar, etc.) con las que existe una convergencia metodológica. En ese caso, se trata de una propuesta que es genuinamente interdisciplinar, en la medida que incorpora contenidos procedentes de varias disciplinas que tienen relación con "conocer" la actividad educativa. Para este caso interdisciplinar, se amplía el radio de acción mediante las contribuciones de otras disciplinas, tales como la Filosofía, la Psicología y la Inteligencia Artificial, además de las aportaciones de la Tecnología.

\footnotetext{
${ }^{7}$ Sin embargo, esto no siempre se asume en Educación, puesto que, en algunos ámbitos, persiste su consideración como un conocimiento subalternado. La subalternación comporta aceptar varios aspectos. (i) Los conceptos que se usan en una Ciencia — en este caso, de la Educación — no tienen una significación intrínseca distinta a los que tienen en las disciplinas generadoras, que serían, fundamentalmente, la Psicología, la Sociología, la Biología y la Antropología. Su sentido y referencia no variarían con respecto a la Educación. (ii) Los principios provenientes de otras disciplinas que se usan en Educación equivalen a principios de intervención pedagógica.

Pero no basta con que las disciplinas generadoras prueben algo para que quede probado pedagógicamente. La Ciencia de la Educación es una disciplina autónoma. Por eso el desarrollo de principios pedagógicos de intervención requiere una elaboración en conceptos con significación intrínseca del ámbito educativo. Cuando las disciplinas autónomas se relacionan entre sí, cada una de ellas puede hacer uso de los conocimientos obtenidos por otras disciplinas para sus investigaciones, pero su uso correcto no garantiza por si solo la validez de los resultados obtenidos en otra disciplina. En este caso se da una dependencia disciplinar, pero no hay subalternación (cfr. Touriñán, 2012).
} 
Como los problemas educativos son problemas complejos, la complejidad — estructural y dinámica, con sus elementos epistemológicos y ontológicos- en las Ciencias de la Educación desafía el tratamiento disciplinar, que surgió para dar cuenta de una realidad que, considerada de una manera unidimensional, estaba orientada por enfoques tradicionales. Esto exige un planteamiento diferente de la manera de organizar el conocimiento y la investigación en el caso de la Educación. La existencia de un pluralismo metodológico está fuera de duda y la Filosofía de la Ciencia no puede ignorar la diversidad metodológica al afrontar los problemas científicos (González, 2012b). En este nuevo contexto, integrar metodológicamente aportaciones disciplinares se ha convertido en algo necesario y su gestión es una demanda cada vez más poderosa de la Ciencia contemporánea. Parece que, sin embargo, la Filosofía de la Ciencia apenas comienza a prestarle atención de manera sistemática (Mäki, 2016, 327).

\subsection{Interdisciplinariedad y multidisciplinariedad}

En gran medida, el desarrollo del conocimiento científico $-\mathrm{y}$ también la innovación tecnológica - se lleva a cabo actualmente mediante la intervención de equipos de trabajo interdisciplinarios. Esto se observa de forma clara en la Educación, puesto que la complejidad de los fenómenos educativos requiere colaboración entre diversas disciplinas, sean disciplinas de carácter pedagógico (Didáctica, Orientación educativa, etc.) o con otra raíz metodológica de origen (Psicología, Sociología, etc.).

Entre los problemas que son objeto de la investigación educativa y que se sitúan en contextos de diferentes disciplinas, cabe señalar los estudios acerca del aprendizaje y las indagaciones sobre la Educación mediada por la Tecnología. Tradicionalmente el foco de la acción educativa se situó en la enseñanza. Al desplazarse ese foco al aprendizaje, las Ciencias de la Educación acogen esa investigación que, aunque siempre estuvo relacionada con este ámbito disciplinar, anteriormente era objeto de estudio de la Psicología. Entre otras disciplinas, involucran a la Sociología, la Economía, la Neurociencia, la Arquitectura y, de forma destacada, a la Tecnología (en especial, a las Tecnologías de la Información y la Comunicación).

Pero el estudio de la educación como objeto de conocimiento tiene sus perfiles propios. Así, no se puede limitar a las explicaciones que, desde otras bases metodológicas, hacen de esos fenómenos. Porque, de ser así, "lo que se ofrece es una sucesión de contribuciones que funcionan al modo de una superposición de capas o bien como una yuxtaposición de elementos procedentes de las contribuciones de diversas disciplinas" (González, 2008a, 54-55). Y los fenómenos educativos tienen características y propiedades diferentes a la simple suma de los 
fenómenos en los que se pueden dividir ${ }^{8}$. Junto a que el todo es más que la suma de las partes, hay que añadir que esos otros enfoques metodológicos pertenecen a otros ámbitos de estudio, de modo que no captan aquello que es central en el objeto de estudio específico de la Ciencia de la Educación: lo genuinamente educativo.

Para que haya interdisciplinariedad, se requiere integración de las contribuciones de varias disciplinas a la hora de afrontar un problema. Supone partes interdependientes de conocimiento, que se relacionan a través de estrategias tales como la parte y el todo, lo particular y lo general, etc. Junto al componente epistemológico está, obviamente, el planteamiento metodológico, orientado desde enfoques colaborativos para combinar la diversidad y la unidad (Stember, 1991). Porque la interdisciplinariedad es un esfuerzo complejo, que busca explicar las relaciones, procesos, valores y contexto en términos de confluencia.

Los estudios de Ciencia, Tecnología y Sociedad (CTS) buscan esa confluencia desde puntos de origen distintos, de modo que pueden presentarse como ejemplo de un trabajo interdisciplinar?. Pero más próximo al caso de las Ciencias de la Educación es la concepción de las Ciencias Cognitivas propuesta por Simon (1980). Se trata de una propuesta que es claramente interdisciplinar, en la medida que incorpora contenidos procedentes de varias disciplinas que tienen relación con el hecho de "conocer", especialmente de la Filosofía, la Psicología y la Inteligencia Artificial (cfr. González, 2008a, 55). Este ejemplo permite ilustrar lo que comúnmente se entiende por esta forma de integración del conocimiento. Más aún, a mi juicio, podría servir como modelo de una Ciencia de la Educación.

Tanto la trayectoria intelectual de Simon como sus propuestas curriculares son habitualmente interdisciplinares. Uno de los rasgos más sobresalientes de su trayectoria es la diversidad de campos en los que trabajó: Ciencia Política, Economía, Psicología, Inteligencia Artificial o Filosofía de la Ciencia. Pero el recorrido por esta variedad de ámbitos temáticos responde a una búsqueda concreta, que constituye el hilo conductor de toda su concepción: el estudio de los procesos de pensamiento que emplean los agentes al tomar decisiones, que fue siempre la preocupación central de Simon. Así, consideraba que se podía diversificar la

\footnotetext{
${ }^{8}$ Antonio Rodríguez lo explica mediante una analogía con los fenómenos químicos, donde al producirse la unión de dos o más elementos, el producto de dicha unión es un fenómeno nuevo, que tiene propiedades y características diferentes a las de los elementos que lo componen, de modo que no vale con conocer los elementos simples para explicar el nuevo elemento, sino que hay que elaborar una nueva explicación para dicho elemento (Rodríguez Martínez, 2006, 35).

${ }^{9}$ En Educación Secundaria, materias como la Cultura científica o Cultura clásica, responden también a este enfoque. Se observa una tendencia a recuperar el enfoque interdisciplinar en decisiones como la recuperación de áreas como "Ciencias de la Naturaleza”, en sustitución de Física y Biología. Sucede que la formación disciplinar de los profesores, a veces, supone un obstáculo. La propuesta verdaderamente interdisciplinar es la propuesta metodológica del trabajo por tareas que, por otra parte, se ve dificultado por la presencia de disciplinas en el currículum.
} 
indagación de la naturaleza de los procesos de pensamiento presentes al decidir, para buscar después la interrelación. Entonces los procesos se podían estudiar en cada uno de los dominios del quehacer humano y lo descubierto en uno de ellos podía arrojar luz sobre los demás (cfr. González, 2003, 7).

Esta concepción de Simon no es resultado de una mera diversidad de enfoques. Su trayectoria ofrece claramente un modelo de trabajo interdisciplinar. Lo hace por la presencia de un eje vertebrador de toda su concepción, que se encuentra en su teoría de la racionalidad limitada (bounded rationality). Porque lo propio del quehacer interdisciplinar es que haya un punto de encuentro (meeting point) de las distintas facetas, de manera que los distintos ángulos convergen en ese lugar compartido. Así lo reconocía el Nobel de Economía, que llegó a describir su carrera como "peripatética": en su autobiografía, al hacer balance de los ámbitos del saber por los que se había interesado - y en los que es sabido que además hizo aportaciones relevantes - cita la Ciencia Política, la Teoría de la Organización, la Economía, la Ciencia de la Gestión, la Informática, la Psicología y la Filosofía de la Ciencia (Simon, 1991, xviii). Pero también señaló que su investigación era, en el fondo, "monotemática" (Simon, 2001, 501-505), lo que resulta indicativo de la interdisciplinariedad.

Para optar en las Ciencias de la Educación por una concepción metodológica en términos de interdisciplinariedad, inciden tanto factores internos a la actividad educativa como externos a ella (estos aspectos pueden, en algunos casos, conducir también a la multidisciplinariedad o la disciplinariedad cruzada). Entre los primeros están los cambios producidos en el plano conceptual y algunos problemas metodológicos relacionados con los fenómenos de aprendizaje. Entre los segundos se encuentran las nuevas demandas sociales o el cumplimiento de requisitos de carácter legal.

La confluencia de estos factores permite entender el surgimiento de un área interdisciplinar como la Educación mediada por la Tecnología. Se trata de estudios que son interdisciplinares en su enfoque metodológico, porque una serie de disciplinas - tales como la Pedagogía, las Ciencias de Internet y la Inteligencia Artificial- convergen en puntos comunes. Desde el punto de vista de contenidos, requieren tanto el manejo de teorías educativas como el uso de conocimientos procedentes de otros ámbitos disciplinares, como son las teorías del aprendizaje, y conocimientos instrumentales que aportan otras disciplinas originalmente no pedagógicas.

Cuando el asunto se analiza desde la Filosofía de la Ciencia, se observa que, que en el campo de las Ciencias de la Educación — al igual que en la Sociología, la Historia o en la Ciencia de la Administración- los investigadores proporcionan a menudo una imagen de interdisciplinariedad que es estrecha o superficial. Así, presentan diagnósticos erróneos y recetas dudosas para promover interdisciplinariedad; los informes que ofrecen pueden carecer de claridad conceptual o profundidad de razonamiento, etc. Por otra parte, pierden a veces versiones 
de interdisciplinariedad que serían fáciles de reconocer para un análisis filosófico-metodológico, posturas que serían útiles para avanzar en este campo (Mäki, 2016, 327). Por eso, es preciso que la Filosofía de la Ciencia ponga también en su agenda el complejo fenómeno de la interdisciplinariedad.

Es un hecho que la interdisciplinariedad está creciendo. Lo hace en paralelo al crecimiento de los enfoques de pluralismo metodológico, que están basados en la diversidad de orientaciones científicas y están de acuerdo en descartar el universalismo metodológico (González, 2012b). Esto es especialmente relevante para los problemas estudiados por la Filosofía de la Ciencia, que debe tomar interés por estos planteamientos. Sucede, además, que esta disciplina está especialmente equipada para abordar esos nuevos enfoques acerca de los fenómenos, sean naturales, sociales o artificiales.

Hay, para Mäki y MacLeod, muchas más variedades de interdisciplinariedad que las sugeridas por las concepciones populares, que tienden a requerir de interacciones que sean integradoras y colaborativas. A su juicio, existen otras formas de interacción efectivas productivas, que se llevan a cabo a través de fronteras disciplinarias donde ni la integración ni la colaboración están sustancialmente involucradas. Considera que puede ser igualmente probable que estimulen la innovación científica y el progreso (Mäki y MacLeod, 2016, 323-324).

Si se sigue la caracterización de la interdisciplinariedad de Mäki, que remite simplemente a cualquier relación relevante entre dos o más disciplinas científicas o sus partes, entonces tenemos diversas situaciones. (i) Disciplinas que están claramente abiertas hacia adentro y tienen una alta propensión a adoptar ítems de otras disciplinas (a mi juicio, la Ciencia de la Educación pertenecería a este primer tipo). (ii) Disciplinas que están más abiertas hacia afuera, de modo que están dispuestas a expandir el alcance de sus propias teorías y procedimientos, para llegar a dominios siempre nuevos. (iii) Disciplinas que están mucho más cerradas y con mentalidad de autosuficientes, por lo que hay mucha menos actividad interdisciplinar transfronteriza (Mäki, 2016, 331).

Respecto a estas formas de interdisciplinariedad, la Filosofía de la Ciencia manifiesta los dos tipos de apertura: hacia adentro y hacia afuera. En general, la Filosofía es quizás la disciplina más abierta de todas. Muestra de facto un esfuerzo intelectual que busca expandirse, sin apenas límites de ningún tipo en cuanto a atender objetos de estudio nuevos y desde ángulos novedosos (como sucede con la Filosofía y Metodología de las Ciencias de lo Artificial). A tenor de este espíritu expansionista, Mäki propone ampliar el dominio de la Filosofía de la Ciencia para incluir la interdisciplinariedad (Mäki, 2016, 333). La urgencia se debe a una doble preocupación: teórica y práctica.

Una preocupación intelectual se refiere al sueño de recuperar la unidad de la Ciencia, que se puede lograr — al menos, en parte- por la vía de la interdisciplinariedad. Considera Mäki que se ha perdido la visión de unidad, debido 
a la progresiva especialización. Esta diversificación metodológica habría llegado demasiado lejos. Otra preocupación — la principal— para la resaltar la importancia de la interdisciplinariedad es de índole práctica: la Ciencia está dividida en disciplinas y subdisciplinas. Pero los problemas más apremiantes del mundo real (desde cambio climático a la pobreza global) no lo están. Por tanto, solo pueden abordarse con éxito cruzando límites disciplinarios.

Otra opción metodológica es la multidisciplinariedad. En 1955 Gordon W. Blackwell hizo su propuesta acerca del trabajo multidisciplinar realizado en equipo. Caracterizó varios tipos de investigación, atendiendo a tres dimensiones, que forman, de hecho, un continuo: a) el número de personas que hacían la investigación, b) los tipos de acciones implicadas en el proceso, y c) el número de disciplinas implicadas. Consideró que un equipo de investigación verdaderamente multidisciplinario debe satisfacer tres condiciones básicas: 1) debe haber más de un investigador involucrado en el proceso de investigación; 2) los investigadores deben representar al menos dos disciplinas distintas; y 3) debe haber trabajo colectivo, al menos en cierto grado, por lo que los investigadores deben trabajar en equipo y no por separado.

Esto supone caracterizar una opción metodológica desde una perspectiva externa, pues no se centra en el estudio de cómo la postura multidisciplinar puede servir para enfocar los problemas, los modelos y los procesos de contrastación en la investigación científica, sino que se orienta expresamente hacia el trabajo en equipo encaminado hacia la multidisciplinariedad. A este respecto, Blackwell traza una línea continua, para representar las distintas tipologías de relación disciplinar. Sitúa en un extremo el investigador solitario, que trabaja en una disciplina. Ubica, en el otro, al equipo investigador multidisciplinario, donde los investigadores de más de una disciplina trabajan de forma colectiva en un problema.

Entre esos dos extremos señalados por Blackwell se encuentran las distintas posibilidades de trabajo en equipo, donde están presentes algunos de los elementos que caracterizan la investigación de tipo interdisciplinar: (i) un investigador solitario que trabaja en más de una disciplina, (ii) dos o más investigadores que trabajan por separado en la misma disciplina, (iii) dos o más investigadores trabajando en equipo en la misma disciplina, y (iv) dos o más investigadores que trabajan por separado en diferentes disciplinas (Blackwell, 1955, 368).

Que el trabajo en equipo sea "interdisciplinar" o "multidisciplinar" depende preferentemente de la perspectiva interna, pues se basa en la orientación metodológica utilizada para hacer la investigación. En el primer caso se busca expresamente el punto de encuentro temático, mientras que, en el segundo caso, prevalecen las propias líneas de investigación seguidas por los científicos (en ese laboratorio o institución científica pública o privada). Así, lo que caracteriza a la investigación multidisciplinar es que o bien meramente yuxtapone los distintos enfoques o bien los superpone como si fuesen "capas" alrededor de un tema de 
investigación; pero no intenta integrar las contribuciones en torno a un eje vertebrador.

A mi juicio, buscar que el conocimiento que ofrece una de esas yuxtaposiciones o capas arroje luz para el problema planteado o refuerce el conocimiento de la otra requiere algún tipo integración. Ese mayor grado de interrelación facilita, además, las oportunidades de evaluar críticamente las ideas que utilizan al reflejarse una disciplina "en el espejo" de la otra. En lo que atañe al desarrollo de la Ciencia de la Educación, la multidisciplinariedad tiene una utilidad limitada, de manera que es útil metodológicamente como punto de contraste. Quizá esto se aprecie mejor si se piensa en la Física y la Música ante el fenómeno del sonido percibido. Porque, para estudiar las perturbaciones mecánicas generadas en el mundo externo, que llegan al oído humano, la Física no tiene que cambiar sus métodos propios. Pero puede ofrecer una visión diferente de la Música como Arte de combinar sonidos y silencios, cuando busca la armonía u otra forma de relación que llame la atención.

Cabe considerar que el currículum de la Educación Secundaria puede servir como ejemplo de multidisciplinariedad, sobre todo en el Bachillerato actual. Diversas disciplinas abordan distintos aspectos de un problema (p. ej., la Ilustración puede estudiarse desde la Filosofía, desde la Literatura, desde la Historia o desde la Historia del Arte) y es el estudiante quien debe integrar las distintas perspectivas en ausencia de un discurso común, que es lo que aportaría la interdisciplinariedad.

\subsection{Transdisciplinariedad y la disciplinariedad cruzada (crossdisciplinarity)}

La transdisciplinariedad se entiende como el nivel metodológico donde se subsumen contenidos procedentes de diversas disciplinas, que pueden ser humanísticas, científicas o tecnológicas. En esa diversidad metodológica se busca un tipo de integración, que está orientado hacia problemas que, de un modo u otro, son nuevos. Esto es lo que sucede con los Estudios de Género, que no solo desean ser teoréticos sobre un ámbito de estudio perfilado en las últimas décadas, sino que buscan, además, de alguna manera, una proyección práctica ante las nuevas circunstancias sociales.

En este sentido, con la transdisciplinariedad se busca la unidad del marco conceptual que vaya más lejos de las específicas perspectivas disciplinarias, de modo que permitan una integración en un nuevo nivel, lograda por vía de subsunción conceptual para afrontar un nuevo problema. La complejidad, tanto estructural como dinámica, aparece entonces de forma muy clara. Por eso, resulta difícil a veces determinar, por ejemplo, si estudios concretos cuyo objeto es el cambio climático o cómo lograr una base científica para una aplicación adecuada de las TIC a la educación son estudios interdisciplinares o pertenecen ya a la categoría 
de lo transdisciplinar. Esto depende de la orientación metodológica seguida por los investigadores en los respectivos casos.

Situados en este plano de análisis filosófico-metodológico, cabe pensar - como hace Max-Neef - que lo ofrecido por la transdisciplinariedad es, en rigor, un modo distinto de ver el mundo, en lugar de propiciar una nueva disciplina o presentar una super-disciplina. Así, la investigación disciplinaria concierne normalmente a un solo nivel de realidad, mientras que la indagación transdisciplinaria extiende su acción a través de varios niveles de realidad (cfr. Max-Neef, 2005, 15).

Tenemos entonces que, en la transdisciplinariedad, hay un uso compartido de conceptos, métodos y argumentos utilizados en terrenos disciplinares muy distintos y que giran en torno a un eje temático. De ahí su transversalidad metodológica. En cambio, en la interdisciplinariedad se conserva la especificidad de las disciplinas y se mantienen sus aportes cognoscitivos y sus planteamientos metodológicos. Porque "la interdisciplinariedad depende de la disciplinariedad, tanto conceptual como causalmente (...) Sin disciplinas, la interdisciplinariedad sería inconcebible. Si las disciplinas se desvanecieran, como algunos entusiastas de la interdisciplinariedad a veces parecen desear, la idea misma de la interdisciplinariedad se volvería inconcebible" (Mäki, 2016, 331). A este respecto, la interdisciplinaridad no se limita a "poner en contacto" las aportaciones que se hacen desde diferentes disciplinas, sino que requiere la elaboración de un discurso compartido.

Otra forma de relación entre disciplinas recibe la denominación de crossdisciplinarity, que aquí se traduce por "disciplinariedad cruzada" ${ }^{0}$. Se usa, en general, para una intersección disciplinar, de modo que, en principio, puede referirse a cualquier actividad que involucre dos o más disciplinas académicas. Pero se asocia habitualmente al hecho de relacionar disciplinas de carácter diverso en su configuración metodológica. Sería el caso de distintos investigadores que, desde ángulos que inicialmente son distantes, trabajan juntos en un mismo tema. Cada uno aporta su conocimiento disciplinario, de modo que ofrecen una diversidad de enfoques metodológicos.

Caben entonces varias opciones desde un punto de vista interno, todas ellas relacionadas metodológicamente con una intersección entre disciplinas. Así, algunos usos de la expresión "disciplinariedad cruzada" sugieren que se concibe

\footnotetext{
${ }^{10}$ La expresión "disciplinaridad cruzada" como traducción de crossdisciplinarity es una opción propia. También lo es el sentido en el que se usa. No se ha encontrado una traducción consensuada para el término crossdisciplinarity. Tampoco hay una acepción fijada. En sentido ordinario, suele aparecer como sinónimo de multidisciplinariedad. Mientras tanto, en la literatura especializada, adquiere significados diversos. Así, a veces designa, de forma genérica, el objetivo de cruzar los límites entre las disciplinas. Pero, en esa acepción, acogería cualquier forma de investigación en la que participen dos o más disciplinas académicas. En ese caso, podría entonces designar tanto la interdisciplinariedad como incluso una forma de integración disciplinar superior a ella.
} 
como una tipología de investigación específica. Se usa cuando se aborda un tema que está fuera del alcance de una disciplina y sin tener, en principio, una integración metodológica con otras disciplinas. Sería el caso del estudio de la Genética, que se entrecruza o tiene intersección con varias disciplinas, tales como los Estudios sociales de la Ciencia. A su vez, la Genética comparte métodos con otras ramas de la Biología, la Química (p. ej., la estructura molecular del ADN), la Ciencia Ambiental (p. ej., Genética de conservación) u otras facetas científicas procedentes de las Ciencias de la Salud, pero sin llegar a integrarse o diluirse en alguna de ellas.

Entendida de una manera estricta, la disciplinaridad cruzada significa que los temas se estudian aplicando enfoques metodológicos de disciplinas no relacionadas. Así entendida, la disciplinariedad cruzada se distingue de la interdisciplinariedad, porque los límites de las disciplinas se cruzan o interseccionan. Pero no lo hacen, en cambio, las propuestas metodológicas ni los supuestos de cada una de las disciplinas involucradas, que sí se combinan en el caso de la interdisciplinariedad. En cierto modo, sería una posición metodológica situada entre la multidisciplinariedad y la interdisciplinariedad.

En este sentido, la Ciencia de la Educación puede estar abierta a la intersección con otras disciplinas, en cualquiera de los tres grupos (Ciencias de la Naturaleza, Ciencias Sociales o Ciencias de lo Artificial). Desde este punto de vista, en consonancia con lo expuesto, cabe pensar lo siguiente: a) la complejidad de los fenómenos educativos justifica la necesidad de superar la especificidad disciplinar, aceptando el "entrecruzamiento disciplinar" como una de ellas; y b) las formas de relación disciplinar que se observan en Educación permiten, además, fórmulas interdisciplinares, multidisciplinares y transdisciplinares.

Cabe afirmar que ámbitos disciplinares como la Sociología de la Educación, la Economía de la Educación, etc., son resultado de esas formas de relación disciplinar. Inicialmente pueden ser una forma de "disciplinariedad cruzada" y, posteriormente, dar lugar a una genuina interdisciplinariedad. Para M. Stember (1991, 4), el nivel más bajo de colaboración disciplinar es la "disciplinariedad cruzada" (crossdisciplinarity), que consiste en la visión de una disciplina desde la perspectiva de otra. Es lo que sucede en el caso de la Física de la Música, en cuanto que se ofrece una interpretación de la Música desde la perspectiva de la Física $^{11}$. Pero, en mi opinión, esto puede suponer un enfoque mucho más inte-

\footnotetext{
${ }^{11}$ Manfred Max-Neef $(2005,6)$ usa el término "pluridisciplinariedad" para referirse a una forma de colaboración entre disciplinas en la que hay ya una integración disciplinar, que no se encuentra en la mera multidisciplinariedad y que, a mi juicio, puede recoger este sentido de la idea de crossdisciplinarity. Pero, como la diferencia entre los prefijos "multi" y "pluri" en castellano es borrosa, el concepto "pluridisciplinalidad" no remite intuitivamente a lo que pretende designar, que es la cooperación entre disciplinas en ausencia de coordinación.
} 
grador o inclusivo entre las disciplinas, propiciar un tipo de integración mayor que la multidisciplinariedad ${ }^{12}$.

En suma, aunque haya una especificidad disciplinar de la Ciencia de la Educación, que se centra en la Pedagogía, existe una interacción con un conjunto de disciplinas que conforman las Ciencias de la Educación desde una perspectiva interdisciplinar. Pero, con frecuencia, la investigación educativa involucra dos o más disciplinas académicas, de modo que puede ser también multidisciplinaria o transdisciplinaria o resultado de una disciplinariedad cruzada. En las últimas dos décadas, el espectro y la complejidad de los problemas que aborda la investigación educativa se ha incrementado notablemente. Además, al tratarse de una Ciencia Aplicada, su solución para los problemas atañe a la práctica. Depende muchas veces de la posibilidad de unificación e integración conceptual y metodológica entre campos disciplinarios específicos y, en ocasiones, incluso de otros que están situados más allá de la esfera académica ${ }^{13}$.

\section{Conclusiones}

La Ciencia de la Educación se ha juzgado, hasta ahora, desde estándares propios de las Ciencias Sociales. Pero sus métodos no son adecuados para abordar la acción educativa en cuanto tal, que es su objeto de estudio específico, aunque sí pueden tratar otros aspectos de la educación. Aquí se reconoce su carácter de Ciencia de Diseńo, un campo del conocimiento científico que no se limita a analizar cómo son las cosas, sino que también prescribe cómo deben ser para alcanzar unos fines buscados.

Como Ciencia Aplicada de Diseño, la Ciencia de la Educación ha de abordar el problema de la complejidad, que puede ser tanto estructural como dinámica. Esa complejidad incide en conocimientos, procesos y valores; además, está presente en la propia realidad investigada. Por tanto, cuando se estudia la complejidad en la Ciencia de la Educación, hay que considerar tanto los aspectos que se manifiestan en la elaboración de los diseños (que atañe a factores epistemológicos y metodológicos) como en lo diseñado (el plano ontológico). Atender a lo ontológico en la investigación científica tiene asimismo repercusiones en aspectos metodológicos y epistemológicos.

Así pues, a la hora de estudiar realidades complejas, desde la Filosofía de la Ciencia se resalta, por una parte, la necesidad de la convergencia de diversos enfoques metodológicos - ya sean naturales, sociales o artificiales- y, por otra parte, se insiste desde el punto de vista de su estatuto científico en contar con las aportaciones de distintas disciplinas. La mayor parte de los problemas de la

\footnotetext{
${ }^{12}$ Sin duda, para fundamentar esa opinión se requeriría una argumentación más extensa, a la que se renuncia porque no se dan las condiciones para que sea posible establecer una tipología.

${ }^{13}$ Koskinen y Mäki (2016) han trabajado sobre la transdisciplinariedad extraacadémica.
} 
realidad educativa no pueden abordarse desde una perspectiva disciplinar. Es necesario habilitar otras opciones metodológicas, como la interdisciplinariedad, la multidisciplinariedad, la transdisciplinariedad o el punto de vista de una disciplinariedad cruzada (crossdisciplinarity). También desde la perspectiva de los estudios de la actividad educativa, se hace referencia a la complejidad de los fenómenos educativos, que consideran que tienen carácter pluriparadigmático, plurimetodológico y multidisciplinar (Arnal, Del Rincón y Latorre, 1994, 36-37).

Cabe, finalmente, hacer una llamada a la Filosofía de la Ciencia para que, en su agenda, incluya una investigación sistemática sobre la Ciencia de la Educación, que esté centrada fundamentalmente en dos cuestiones: (i) su carácter de Ciencia de Diseño y (ii) los aspectos filosóficos de la interdisciplinariedad en la investigación educativa, donde la interdisciplinariedad se entienda en sentido genérico, esto es, como cualquier relación relevante entre dos o más disciplinas científicas o sus partes (Mäki, 2016).

\section{Referencias bibliográficas}

Aristóteles (1985). Ética Nicomaquea. Madrid: Gredos.

Arnal, Justo, Del Rincón, Delio y Latorre, Antonio (1994). Investigación Educativa. Fundamentos y Metodología. Barcelona: Labor.

Blackwell, Gordon W. (1955). Multidisciplinary Team Research. Social Forces, 33(4), 367-374.

González, Wenceslao J. (2003). Herbert A. Simon: Filósofo de la Ciencia y economista (1916-2001). En Wenceslao J. González (ed.), Racionalidad, historicidad y predicción en Herbert A. Simon (pp. 7-63). A Coruña: Netbiblo.

González, Wenceslao J. (2007). Configuración de las Ciencias de Diseño como Ciencias de lo Artificial: Papel de la Inteligencia Artificial y de la racionalidad limitada. En Wenceslao. J. González (ed.), Las Ciencias de Diseño: Racionalidad limitada, predicción y prescripción (pp. 41-69). A Coruña: Netbiblo.

González, Wenceslao J. (2008a). El enfoque cognitivo en la Ciencia y el problema de la historicidad: Caracterización desde los conceptos. Letras, 79(114), 51-80.

González, Wenceslao J. (2008b). La televisión interactiva y las Ciencias de lo Artificial. En María José Arrojo Baliña, La configuración de la televisión interactiva: De las plataformas digitales a la TDT (pp. xi-xvii). A Coruña: Netbiblo.

González, Wenceslao J. (2012a). Las Ciencias de Diseño en cuanto Ciencias de la Complejidad: Análisis de la Economía, Documentación y Comunicación. En Wenceslao J. González (ed.), Las Ciencias de la Complejidad: Vertiente dinámica de las Ciencias de Diseño y sobriedad de factores (pp. 7-30). A Coruña: Netbiblo.

González, Wenceslao J. (2012b). Methodological Universalism in Science and its Limits: Imperialism versus Complexity. En K. Brezechczyn y K. Paprzycka (eds.), 
Thinking about Provincialism in Thinking, Poznan Studies in the Philosophy of the Sciences and the Humanities (vol. 100, pp. 155-175). Ámsterdam: Rodopi.

González, Wenceslao J. (2013). The Roles of Scientific Creativity and Technological Innovation in the Context of Complexity of Science. En Wenceslao J. González (ed.), Creativity, Innovation, and Complexity in Science (pp. 11-40). A Coruña: Netbiblo.

González, Wenceslao J. (2015). Philosophico-Methodological Analysis of Prediction and its Role in Economics. Dordrecht: Springer.

González, Wenceslao J. (2017). Cambio conceptual y diversidad científica: El papel de la historicidad en la dinámica de la Ciencia. Factótum. Revista de Filosofía, 18, 10-32. Disponible en: http://www.revistafactotum.com

González, Wenceslao J. (2018). Internet en su vertiente científica: Predicción y prescripción ante la complejidad. ArtefaCToS. Revista de estudios de la Ciencia y la Tecnologia, 7(1), 75-97. https://dx.doi.org/10.14201/art2018717597

Koskinen, Inkeri y Mäki, Uskali (2016). Extra-academic transdisciplinarity and scientific pluralism: what might they learn from one another? European Journal for Philosophy of Science, 6(3), 419-444. https://dx.doi.org/10.1007/s13194-0160141-5

Mäki, Uskali (2016). Philosophy of interdisciplinarity. What? Why? How? European Journal for Philosophy of Science, 6(3), 327-342. https://dx.doi.org/10.1007/ s13194-016-0162-0

Mäki, Uskali y Macleod, Miles (2016). Interdisciplinarity in action: Philosophy of Science perspectives. European Journal for Philosophy of Science, 6(3), 323-326. https://dx.doi.org/10.1007/s13194-016-0161-1

Max-Neef, Manfred A. (2005). Foundations of transdisciplinarity. Ecological Economics, 53(1), 5-16. Disponible en: http://aoatools.aua.gr/pilotec/files/bibliography/ transdiscipl_MaxNeef_ecolEcon3345059585/transdiscipl_MaxNeef_ecolEcon.pdf

Niiniluoto, Ilkka (1993). The Aim and Structure of Applied Research. Erkenntnis, $38(1), 1-21$.

Quintana Cabanas, José María (1983). Pedagogía, Ciencia de la Educación y Ciencias de la Educación. En José Basabe Barcala (ed.), Estudios sobre Epistemología y Pedagogía (pp. 75-107). Madrid: Anaya.

Rodríguez Martínez, Antonio (2006). Conocimiento de la educación como marco de interpretación de la Teoría de la Educación como disciplina. Tendencias Pedagógicas, 11,31-53. Disponible en: https://dialnet.unirioja.es/descargalarticulo/2089360.pdf

Simon, Herbert A. (1980). Cognitive Science: The Newest Science of the Artificial. Cognitive Science, 4, 33-46.

Simon, Herbert A. (1991). Models of my Life. New York, NY: Haper Collins-Basic Books.

Simon, Herbert A. (1996). The Sciences of the Artificial, $3^{\text {a }}$ ed., Cambridge, MA: The MIT Press. 
La Ciencia de la Educación ante las diversas opciones metodológicas de índole disciplinar

Simon, Herbert A. (2001). On Simulating Simon: His Monomania, and its Sources in Bounded Rationality. Studies in History and Philosophy of Science, 32(3), 501-505.

Stember, Marilyn (1991). Advancing the Social Sciences Through the Interdisciplinary Enterprise. The Social Science Journal, 28(1), 1-14.

Tourinán, José Manuel (1987). Teoría de la Educación. (La educación como objeto de conocimiento). Madrid: Anaya.

Touriñán, José Manuel (2012). Estudiar e investigar: Una aproximación desde la perspectiva conceptual. La autonomía funcional y la complementariedad metodológica como principios de investigación pedagógica. En José María Touriñán y Rafael Saez Alonso (eds.), Teoría de la Educación, Metodología y focalizaciones. La mirada pedagógica (pp. 204-263). Netbiblo: A Coruña. 